\title{
The Effect of Trimebutine and/or Helicobacter pylori Eradication on the Gastroesophageal Reflux Disease, Irritable Bowel Syndrome, and Functional Dyspepsia Overlapping Disorders
}

TO THE EDITOR: Hussain et al ${ }^{1}$ recently investigated the trimebutine effect on the model of overlap syndrome (OS) in guinea pigs; they concluded that trimebutine is effective on upper and lower gastrointestinal tract (GIT) motor function in a peripheral corticotropin-releasing factor induced OS model, thereby signifying the trimebutine introduction for the treatment of OS between functional dyspepsia (FD) and irritable bowel syndrome (IBS) patients.

Although current information regarding the overlapping gastroesophageal reflux disease (GERD), IBS, and FD is limited, existing evidence supports the potential concomitance of GERD with IBS and FD in the same patients, thereby signifying the diverse treatments introduction that could impact on the healthrelated quality of life (HR-QOL) of these patients; ${ }^{2}$ as mentioned by the authors, ${ }^{1}$ such overlaps worsened HR-QOL. Moreover, Helicobacter pylori infection may also contribute to the pathophysiology of GERD, at least in certain ethnic populations, and FD and/ or IBS; ${ }^{3,4}$ in IBS, the existence of FD is connected with $H$. pylori infection and perceived stress. ${ }^{4}$

Our own preliminary data, ${ }^{5}$ revealed that $H$. pylori infection is frequent in patients with GERD-IBS-FD and/or erosive esophagitis and $H$. pylori eradication plus proton pump inhibitors and/ or trimebutine regimens offer improvement of HR-QOL predominantly in patients treated with trimebutine. Consequently, we confirmed our preliminary data, ${ }^{6}$ thereby signifying the trimebutine efficacy in the aforementioned overlapping populations.

Specifically, $H$. pylori-induced mast cells (MCs) are significant effectors of the GIT-brain axis that translate the stress signals into the induction of variable neurotransmitters and pro-inflammatory mediators that might contribute to GIT pathophysiology; $H$. pylori stimulated chronic perceived stress results in decreased host defense and initiates intestinal inflammation through MC-dependent mechanisms, thereby signifying the activation of peripheral cortico- tropin-releasing factor receptors and $\mathrm{MC}$ as significant mechanisms involved in stress linked GIT pathophysiology; ${ }^{7,8} H$. pylori-related stress leads to the induction of IBS, GERD, and additional GIT disorders; ${ }^{8}$ H. pylori-induced MCs activation and mediator release contribute to the development of major IBS and GERD symptoms; ${ }^{7}$ changes in the upper and lower GIT permeability, motility, and secretion can appear, for example, in IBS, and many of these disturbances disappear after $H$. pylori eradication with concomitant improvement of HR-QOL; ${ }^{7}$ and $\mathrm{H}$. pylori-induced disturbed gastric emptying and FD symptoms, are improved after $H$. pylori eradication in FD patients. ${ }^{7}$

In overlapping disorders, apart from several trimebutineinduced effective actions mentioned by the authors, ${ }^{1}$ this drug could also act by: shortening the lag period (ie, time period before constant gastric emptying onset) $;^{5}$ and as a possible antimicrobial agent against GIT bacteria that trigger post-infectious functional gastrointestinal disorders, ${ }^{9,10}$ thereby necessitating further relative research.

Jannis Kountouras, ${ }^{1 *}$ Emmanouel Gavalas, ${ }^{1}$ Michael Doulberis, ${ }^{1,2}$ Stergios A Polyzos, ${ }^{1,3}$ Apostolis Papaefthymiou, ${ }^{1,4}$ Maria Touloumtzi, ${ }^{1}$ Elisabeth Vardaka, ${ }^{1}$ Konstantinos Kountouras, Katerina Papanikolopoulou, ${ }^{1}$ and Panagiotis Katsinelos ${ }^{1}$ 'Department of Medicine, Second Medical Clinic, Aristotle University of Thessaloniki, Ippokration Hospital, Thessaloniki, Macedonia, Greece; ${ }^{2}$ Department of General Internal Medicine, University Hospital Inselspital, Bern, Switzerland; ${ }^{3}$ First Departments of Pharmacology and Medicine, Aristotle University of Thessaloniki, Ippokration Hospital, Thessaloniki, Macedonia, Greece; and ${ }^{4}$ Department of Gastroenterology, 401 General Military Hospital of Athens, Athens, Greece

1. Hussain Z, Jung DH, Lee YJ, Park H. The Effect of Trimebutine on the ioverlap syndrome model of guinea pigs. J Neurogastroenterol Motil 2018;24:669-675.

2. de Bortoli N, Tolone S, Frazzoni M, et al. Gastroesophageal reflux disease, functional dyspepsia and irritable bowel syndrome: common over- 
lapping gastrointestinal disorders. Ann Gastroenterol 2018;31:639-648.

3. Kountouras J, Zavos C, Polyzos SA, Katsinelos P. Helicobacter pylori infection and gastroesophageal reflux disease - Barrett's esophagus sequence "dilemma”. Ann Gastroenterol 2015;28:153.

4. Su YC, Wang WM, Wang SY, et al. The association between Helicobacter pylori infection and functional dyspepsia in patients with irritable bowel syndrome. Am J Gastroenterol 2000;95:1900-1905.

5. Kountouras J, Chatzopoulos D, Zavos C, Boura P, Venizelos J, Kalis A. Efficacy of trimebutine therapy in patients with gastroesophageal reflux disease and irritable bowel syndrome. Hepatogastroenterology 2002;49:193-197.

6. Kountouras J, Zavos C, Chatzopoulos D. H. pylori infection and reflux oesophagitis. Gut 2004;53:912.

7. Kountouras J, Boziki M, Polyzos SA, Katsinelos P, Gavalas E, Zeglinas $\mathrm{C}$, et al. The emerging role of Helicobacter pylori-induced metabolic gastrointestinal dysmotility and neurodegeneration. Curr Mol Med
2017;17:389-404.

8. Kountouras J, Polyzos SA, Deretzi G. Multiple bidirectionality brain-gut interactions in patients with inflammatory bowel disease. Gastroenterology 2018;155:1651-1652.

9. Kountouras J, Sofianou D, Gavalas E, et al. Trimebutine as a potential antimicrobial agent: a preliminary in vitro approach. Hippokratia 2012;16:347-349.

10. Kruszewska H, Zareba T, Tyski S. Examination of antimicrobial activity of selected non-antibiotic drugs. Acta Pol Pharm 2004;61(suppl):18-21.

Financial support: None.

Conflicts of interest: None.

Author contributions: All authors contributed in planning the study, collecting and interpreting data, and in the drafting of the manuscript. 